\title{
Treatment of ligature-induced peri-implantitis defects by regenerative procedures. Part II: A histometric study in dogs
}

\author{
M. A. N. Machado, C. M. Stefani, E. A. Sallum, A. W. Sallum, \\ V. A. Tramontina, G. R. Nogueira-Filho and F. H. Nociti Jr. \\ Department of Prosthesis and Periodontology, University of Campinas, \\ Av. Limeira 901, CEP 13414-018, Piracicaba SP, Brazil
}

(Received 6 December 1999 and accepted 5 September 2000)

\begin{abstract}
The purpose of this study was to evaluate, by histometric analysis, re-osseointegration following treatment of ligature-induced peri-implantitis in dogs. Five dogs were used in this study. Their mandibular premolars (P2, P3 and P4) were first removed. After 3 additional months of healing, two titanium implants were placed on each side of the mandible. After 3 months, the abutment connection was performed and experimental peri-implantitis was induced by placing cotton ligatures in a submarginal position. Ligatures and abutments were removed after one month and the peri-implant bone defects were randomly assigned to one of the treatments: debridement, debridement plus guided-bone regeneration, debridement plus mineralized-bone graft, and debridement plus guidedbone regeneration associated with mineralized-bone graft. Five months post-treatment, the degree of bone contact with the implant surface and the bone area within the threads were measured in 12 threads, the 6 most coronal at each side of each implant. One-way analysis of variance did not reveal statistically significant differences between the treatment modalities $(p>0.05)$. Within the limits of the present study, it can be concluded that there is a limited possibility of reosseointegration around implant surfaces previously exposed by ligature-induced peri-implantitis. (J. Oral Sci. 42, 163-168, 2000)
\end{abstract}

Correspondence to Dr. Francisco Humberto Nociti Junior, Faculdade de Odontologia de Piracicaba/UNICAMP, Av. Limeira, 901 CEP: 13414-018, Piracicaba - S.P. , Brasil
Key words: peri-implantitis; regenerative procedures; re-osseointegration.

\section{Introduction}

Although longitudinal studies have documented the long-term predictability of osseointegrated implants (1), a significant number of complications have been reported (2). Late implant failure has been found to be related to bacterial infection and/or biomechanical overload $(3,4)$. Peri-implantitis is described as an alteration of the tissues around the implant that is observed during the implant maintenance phase and is associated with clinical aspects of periodontitis. Some modalities of treatment have been evaluated around peri-implant bone defects resulting from peri-implantitis: antimicrobial, resective and regenerative therapy $(5,6)$. However, only a few histologic studies concerning the regenerative potential (re-osseointegration) of the treatment modalities around the previously contaminated implant surface are reported, and these studies are not conclusive (5, 7-11). Therefore, the purpose of the present study was to evaluate, by histometric analysis, the re-osseointegration process following the treatment of experimentally ligature-induced peri-implantitis using guided-bone regeneration (PTFE membrane - NAPIO, Bauru, São Paulo, Brazil), alone or in combination with a mineralized-bone graft (Bio-Oss ${ }^{\circledR}$ - Osteohealth Co., New York, USA).

\section{Materials and Methods}

Five 2-year-old mongrel dogs were used in this study. The animals were premedicated with $1.5 \mathrm{ml} / 10 \mathrm{~kg}$ of 
acepromazine. Surgical anesthesia was obtained by intravenous injection of $25 \%$ sodium thiopental solution $(0.5 \mathrm{ml} / \mathrm{kg})$ and supplemented with local administration of $2 \%$ xylocaine (1:50,000 epinephrine). At the beginning of the experiment, all mandibular premolars were removed. After 3 months of healing, full-thickness flaps were elevated and two commercially available pure titanium implants (Napio System ${ }^{\circledast}$; Napio, Bauru, São Paulo, Brazil), 8.5 $\mathrm{mm}$ in length and $3.75 \mathrm{~mm}$ in diameter, were placed on each side of the mandible, followed by the repositioning and suturing of the mucoperiosteal flaps.

Three months later, the mucoperiosteal flaps were elevated and the titanium abutments were positioned. Two weeks after the abutment connection, cotton ligatures were placed in a submarginal position around the abutments and the dogs were fed a soft diet to promote plaque accumulation and induce peri-implant inflammation with loss of bone. After 1 month of plaque accumulation, a significant inflammation could be seen at the peri-implant mucosa and bone loss was clinically and radiographically detected. At this time, the ligatures were removed, and a plaque-control regime was initiated (hygienic phase) consisting of daily brushing and topical application of $0.12 \%$ chlorhexidine gluconate. In addition, systemic administration of metronidazole hydrochloride (250 $\mathrm{mg}$ /day) was established for 3 weeks.

Two weeks after the beginning of the hygienic phase, full-thickness flaps were elevated. The abutments were removed and the granulation tissue around the implants was carefully removed using teflon hand curettes. The implant surface was treated with an air-powder abrasive instrument (Profi I ${ }^{\oplus}$; Dabi Atlante, Ribeirão Preto - SP, Brazil) for 30 seconds.

The defects were randomly assigned for one of the following treatments:

1. Debridement: DE

2. Debridement plus guided-bone regeneration: GBR (PTFE membrane -NAPIO, Bauru, SP, Brazil)

3. Debridement plus mineralized-bone graft: BG (BioOss $^{\circledR}$, Osteohealth Co., New York, USA).

4. Debridement plus GBR and BG: GBR/BG

The flaps were repositioned and sutured, the systemic metronidazole administration $\left(\right.$ Flagyl $^{\oplus}$ ) was continued for the following week, and $0.12 \%$ chlorexidine gluconate spray was topically applied for the next 5 months. After a healing period of 4 months, a flap was reflected and the PTFE membranes were removed. Five months after the treatment procedures, i.e., 1 month after the surgical procedure for removing the membranes, the animals were sacrificed. Undecalcified sections were obtained in a bucco-lingual direction and prepared as previously described (12). Subsequently, the sections were stained by toluidine blue stain. The percentage of bone to implant contact and bone area within the 12 most coronal threads of the implant, i.e., 6 threads on each side of each implant, were measured using an image analysis system (KS 400 2.0 $0^{\oplus}$ Kontron Eletroniks, Muchen, Germany) (Fig. 1). In

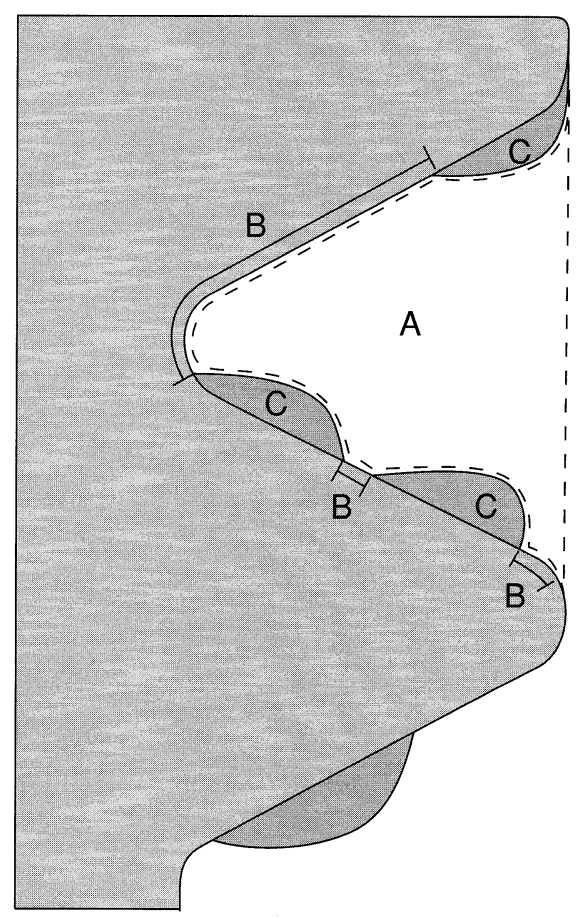

Fig 1. Schematic illustration of the histometric parameters evaluated. In each thread (1 through 6 ) on each side of the implant, the percentage of bone area and bone to implant contact were measured. A: represents the bone area within the limits of a thread of the implant and $\mathrm{B}$ : represents the extension of bone to implant contact within the limits of a thread and C: represents the bone marrow spaces. [adapted from Sennerby et al. (13)]

order to obtain the percentage of bone to implant contact, the extension of the bone in direct contact with the implant surface was measured and divided by the total extension of its corresponding thread, and the value was multiplied by 100 . The procedure to obtain the percentage of bone area within the limits of the thread of the implant was performed as follow: the total area of bone within the limits of each thread of the implant was measured and divided by the total area of its corresponding thread, and this value was multiplied by 100 . The values obtained for 
each of the twelve threads of each implant were pooled, and a mean for each implant was obtained to allow statistical analysis. The experimental design (complete randomized block design) provided a total of 20 implants for statistical evaluation. One-way analysis of variance (ANOVA, $\alpha=$ 0.05 ) was then performed to test the hypothesis that there were no differences between the treatments considering the mean percentage of bone contact and bone area within the threads of the implant.

\section{Results}

Clinical signs of peri-implant inflammation, i.e., redness and suppuration, were drastically reduced after 2 weeks of plaque control and systemic antimicrobial administration. Exposition of the membrane was observed only for one site $(\mathrm{GBR} / \mathrm{BG}) 14$ weeks after placing the regenerative material. The membrane was not removed and the regime of plaque control was maintained until the end of the experimental period. At the time of the sacrifice of the animals, i.e., 5 months after healing, a newly regenerated tissue around the implants was evident for all treatments proposed as previously reported (14). Histometrically, intergroup analysis did not reveal significant differences $(p>0.05)$ among the treatments when considering the degree of bone contact and the bone area within the limits of the exposed implant surface (Figs. 2-7). The mean percentage of bone contact to the implant surface was $26.86 \pm 13.21 ; 30.74 \pm 19.71 ; 28.12 \pm 23.38 ; 27.18 \pm 6.95$ for DE, GBR, BG and GBR/BG, respectively (Table 1). With regard to the bone area within the threads of the implant, the mean percentage was $49.52 \pm 22.82 ; 51.51$ $\pm 18.55 ; 55.74 \pm 21.06 ; 51.50 \pm 10.78$ for DE, GBR, BG and GBR/BG, respectively (Table 1).

\section{Discussion}

Examinations performed during the course of the present study revealed that 1) an inflammatory reaction in the peri-implant mucosa associated with a crater-like circunferential bone defect was the result of submarginal ligature placement and plaque accumulation on the implant surface and 2) it was demonstrated for the proposed treatments that some degree of bone regrowth is possible, however only part of this regenerated bone is in contact with the previously-exposed implant surface. The observation of a reduced amount of re-osseointegration in the present investigation is consistent with most of the literature on this topic. Jovanovic et al. (8) and Singh et al. (15) used antimicrobial therapy associated with an airpowder device to "treat" the contaminated implant surface. The implants were subsequently submerged and protected with membranes. In both studies, a limited amount of re-

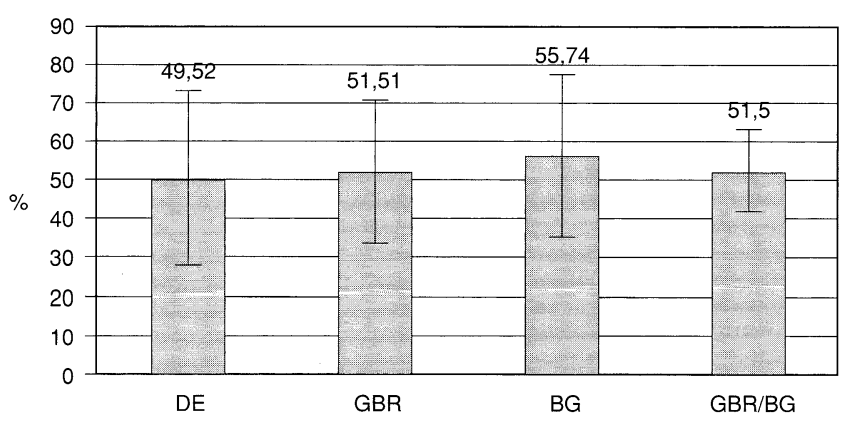

Fig 2. Comparisons of the mean bone area among treatments. DE: flap debridement; GBR: debridement plus guidedbone regeneration; $\mathrm{BG}$ : debridement plus mineralizedbone graft; GBR/BG: debridement plus guided-bone regeneration and mineralized-bone graft.

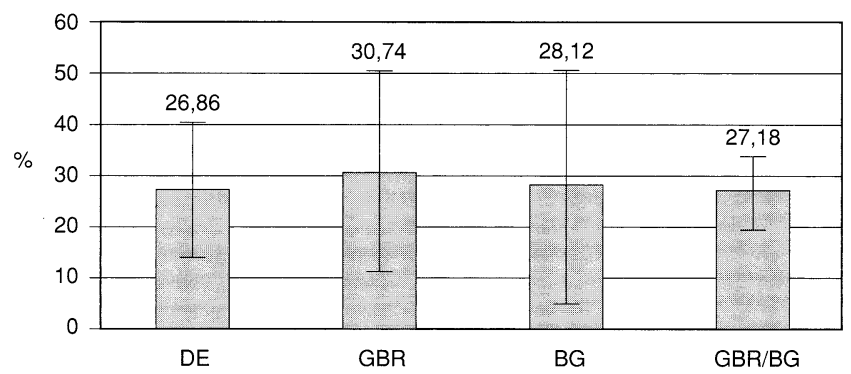

Fig 3. Comparisons of the mean bone contact to the implant surface among treatments. DE: flap debridement; GBR: debridement plus guided-bone regeneration; $\mathrm{BG}$ : debridement plus mineralized-bone graft; GBR/BG: debridement plus guided-bone regeneration and mineralized-bone graft.

Table 1 Bone area (BA) and bone contact (BC) within the limits of the 12 most coronal threads of the implant (mean and standard deviations are presented)

\begin{tabular}{ccc}
\hline DE & BA (\%) & BC (\%) \\
GBR & $49.52 \pm 22.82$ & $26.86 \pm 13.21$ \\
BG & $51.51 \pm 18.55$ & $30.74 \pm 19.71$ \\
GBR/BG & $55.74 \pm 21.06$ & $28.12 \pm 23.38$ \\
& $51.50 \pm 10.78$ & $27.18 \pm 6.95$ \\
& $p=0.960$ & $p=0.983$ \\
\hline
\end{tabular}

osseointegration was reported. The findings of the current study are also in agreement with the results presented by Hanisch et al. (9) who evaluated the re-osseointegration process around implant surfaces in which peri-implantitis had been induced. The authors used collagen sponge 


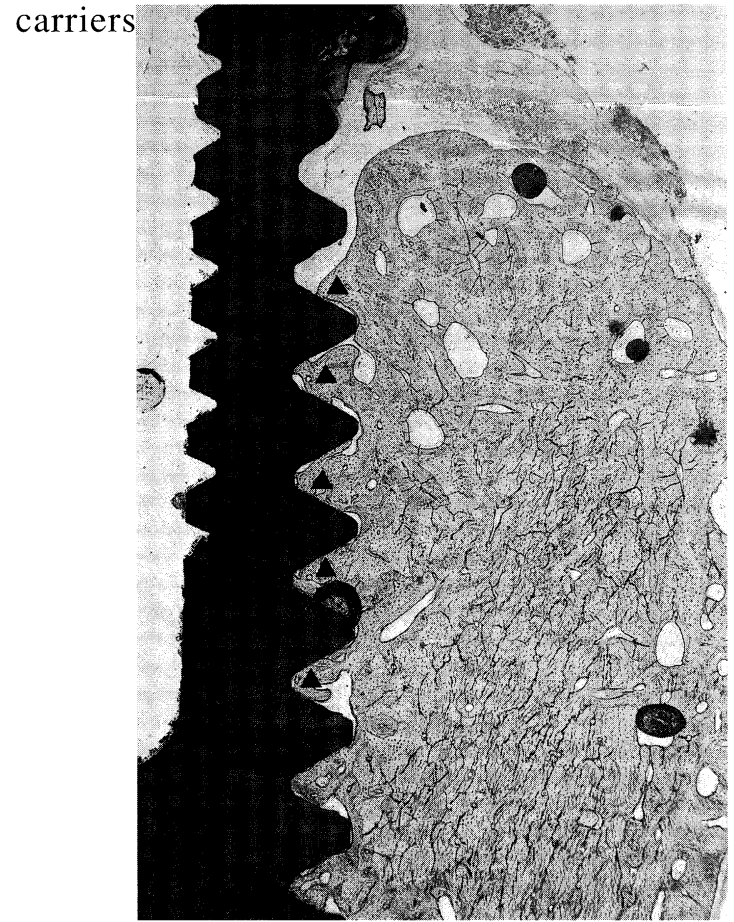

Fig 4. Photomicrograph of a ground section of a submerged implant illustrating new bone $(\boldsymbol{\Delta})$ around the threads of the implant after debridement $(\times 25$ - toluidine blue $)$.

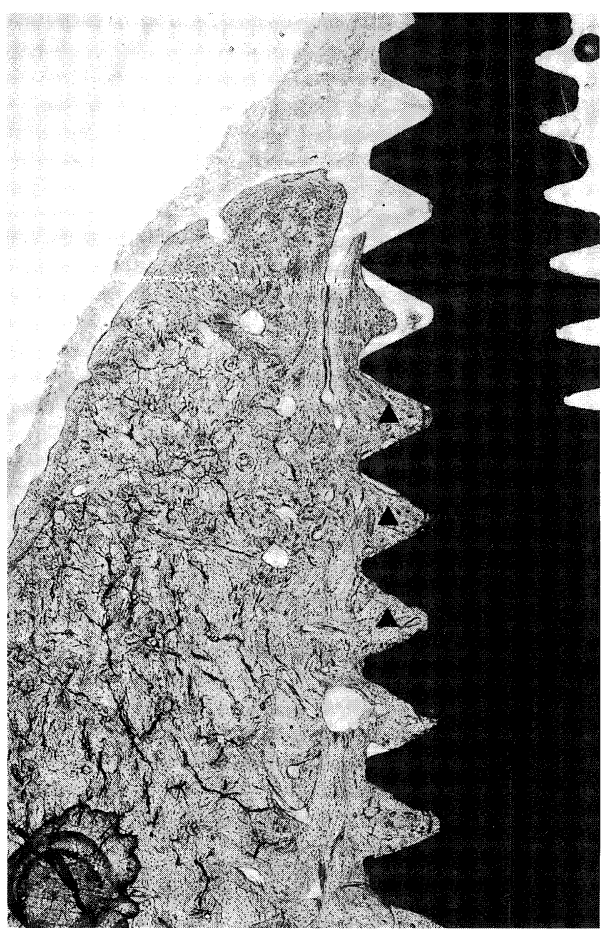

Fig 6. Photomicrograph of a ground section of a submerged implant illustrating new bone $(\boldsymbol{\Delta})$ around the threads of the implant after debridement associated to $\mathrm{BG}$ $(\times 25$ - toluidine blue).

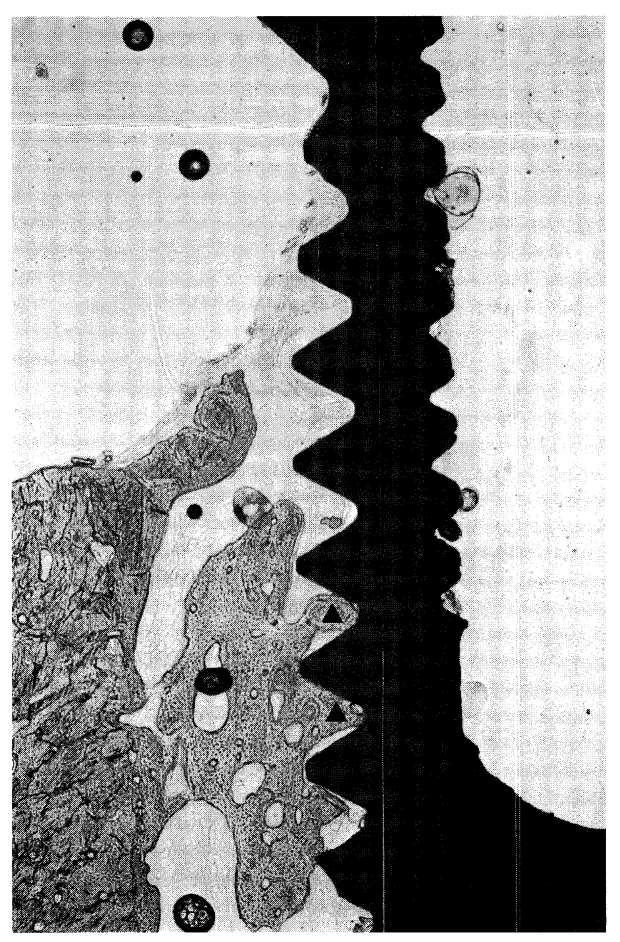

Fig 5. Photomicrograph of a ground section of a submerged implant illustrating new bone $(\boldsymbol{\Delta})$ around the threads of the implant after debridement associated to GBR $(\times 25$ - toluidine blue).

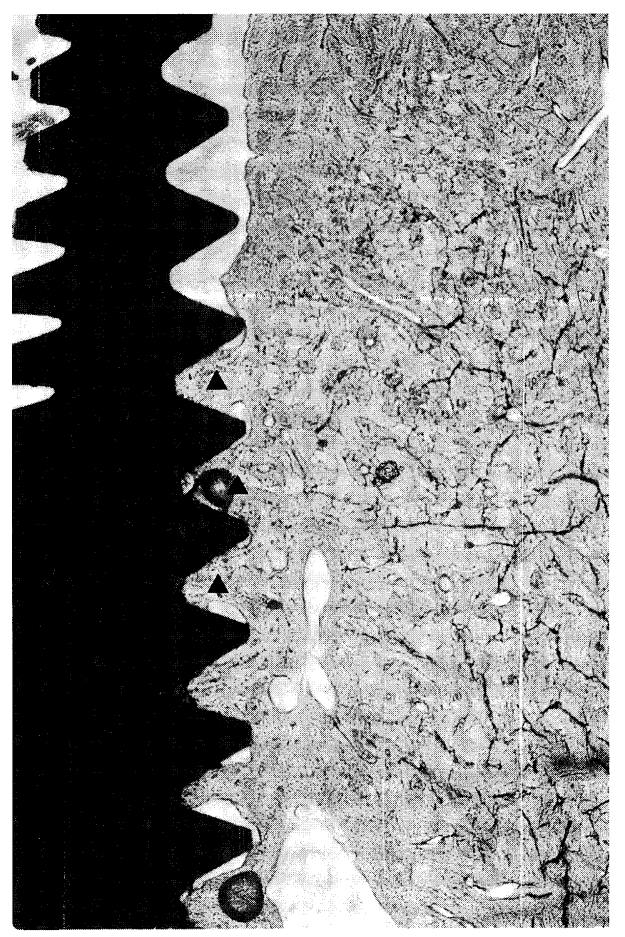

Fig 7. Photomicrograph of a ground section of a submerged implant illustrating new bone $(\boldsymbol{\Delta})$ around the threads of the implant after debridement associated to GBR and BG $(\times 25$ - toluidine blue $)$. 
associated or not to recombinant human bone morphogenetic protein- 2 around the implants. After 4 months of healing, a limited amount of re-osseointegration was observed (25\%). Although Persson et al. (11) reported a significant amount of bone defect fill, the rate of reosseointegration around the previously exposed implant surface was approximately $11 \%$. Wetzel et al. (10) could not determine the reasons for the relatively modest amount of re-osseointegration (2-20\%) encountered in that study. In the present study, a rate from $26 \%$ to $30 \%$ of reosseointegration was observed around the exposed surface of the implants. In contrast to the findings of the above mentioned studies, and also the present investigation, Hürzeler et al. (7) found a substantial amount of reosseointegration after treating ligature-induced periimplantitis using a combination of membranes and bone grafts (a mean of $62 \%$ for test groups and $9 \%$ for the control group). Bone defect morphology and membrane exposure have been indicated as the cause of the poor results, however at the moment, well controlled studies evaluating the variables which would interfere in the process of re-osseointegration are not available. Machado et al. (14) carried out the clinical part of the present study. Clinically, it was concluded that GBR, BG and the combination of the two techniques could enhance the hard-tissue fill of the bone defects caused by peri-implantitis when compared to debridement alone. Nevertheless, the histologic analysis of those specimens revealed that only part of this regenerated bone was re-osseointegrated. Recently, Krozer et al. (16) demonstrated that some procedures used for local disinfection of the exposed implant surface could alter the reactive superficial titanium oxide. Since the biocompatibility of the titanium is advocated for its superficial characteristics, one could suggest that the altered surface would be responsible for the impaired re-osseointegration capacity observed by some investigators, instead of a partially disinfected surface. As a consequence, concern arises with regard to a potential local antimicrobial procedure that would not interfere with the surface properties of the titanium. In contrast to some reports (10), in the present study the exposed membrane was maintained in place and a rigorous plaque control regimen was used. The exposed site was also examined by histometric analysis, that tended to demonstrate negative effects on the evaluated histometric parameters. The difficulty in maintaining the membranes submerged and the impaired results after exposing was previously reported $(5,8)$.

In conclusion, although some degree of bone regrowth of the defect for all modalities of treatment was observed, the amount of re-osseointegration around the exposed surface of the implant was partial. This finding should be considered with caution since the present study have included a small sample size.

\section{Acknowledgments}

This research project was supported by FAPESP - Brasil, Grants no. 97/13213-8 and 98/05045-0. The authors greatly appreciated the assistance of NAPIO for supplying the implants.

\section{References}

1. Albrektsson, T., Zarb, G., Worthington, P. and Eriksson, A.R. (1986) The long-term efficacy of currently used dental implants: a review and proposed criteria for success. Int. J. Oral Maxillofac. Implants 1, 11-25

2. Worthington, P., Bolender, C.L. and Taylor, T.D. (1987) The Swedish system of osseointegrated implants: problems and complications encountered during a 4-year trial period. Int. J. Oral Maxillofac. Implants 2, 77-84

3. Mombelli, A., van Oosten, M.A.C., Schurch, E. and Lang, N.P. (1987) The microbiota associated with successful or failing osseointegrated titanium implants. Oral Microbiol. Immunol. 2, 145-151

4. Quirynen, M., Naert, I. and van Steenberghe, D. (1992) Fixture design and overload influence marginal bone loss and fixture success in the Bränemark system. Clin. Oral Implants Res. 3, 104111

5. Grunder, U., Hürzeler, M.B., Schüpbach, P. and Strub, J.R. (1993) Treatment of ligature-induced peri-implantitis using guided tissue regeneration: a clinical and histologic study in the beagle dog. Int. J. Oral Maxillofac. Implants 8, 282-293

6. Hürzeler, M.B., Quiñones, C.R., Morrison, E.C. and Caffesse, R.G. (1995) Treatment of periimplantitis using guided bone regeneration and bone grafts, alone or in combination, in beagle dogs. Part 1: Clinical findings and histologic observations. Int. J. Oral Maxillofac. Implants 10, 474-484

7. Hürzeler, M.B., Quiñones, C.R., Schüpbach, P., Morrison, E.C. and Caffesse, R.G. (1997) Treatment of peri-implantitis using guided bone regeneration and bone grafts, alone or in combination, in beagle dogs. Part 2: Histologic findings. Int. J. Oral Maxillofac. Implants 12, 168-175

8. Jovanovic, S.A., Kenney, E.B., Carranza, F.A.Jr. and Donath, K. (1993) The regenerative potential of plaque -induced peri-implant bone defects treated by a submerged membrane technique: an 
experimental study. Int. J. Oral Maxillofac. Implants $8,13-18$

9. Hanish, O., Tatakis, D.N., Boskovic, M.M., Rohrer, M.D. and Wikesjö, U.M.E. (1997) Bone formation and reosseointegration in peri-implantitis defects following surgical implantation of rhBMP-2. Int. J. Oral Maxillofac. Implants 12, 604-610

10. Wetzel, A.C., Vlassis, J., Caffesse, R.G., Hämmerle, C.H.F. and Lang, N.P. (1999) Attempts to obtain re-osseointegration following experimental periimplantitis in dogs. Clin. Oral Implants Res. 10, 111119

11. Persson, L.G., Araújo, M.G., Berglundh, T., Gröndahl, K. and Lindhe, J. (1999) Resolution of peri-implantitis following treatment. An experimental study in the dog. Clin. Oral Implants Res. 10, 195-203

12. Donath, K. and Breuner, G.A. (1982) A method for the study of undecalcified bones and teeth with attached soft tissues. The Sage-Schliff (sawing and grinding) technique. J. Oral Pathol. 11, 318-326

13. Sennerby, L., Thomsen, P. and Ericson, L.E. (1992) A morphometric and biomechanic comparison of titanium implants inserted in rabbit cortical and cancellous bone. Int. J. Oral Maxillofac. Implants 7, $62-71$

14. Machado, M.A.N., Stefani, C.M., Sallum, E.A., Sallum, A.W., Tramontina, V.A. and Nociti, F.H. Jr. (1999) Treatment of ligature-induced peri-implantitis defects by regenerative procedures: a clinical study in dogs. J. Oral Sci. 41, 181-185

15. Singh, G., O'Neal, R.B., Brennan, W.A., Strong, S.L., Horner, J.A. and van Dyke, T.E. (1993) Surgical treatment of induced peri-implantitis in the micro pig: clinical and histological analysis. J. Periodontol. 64, 984-989

16. Krozer, A., Hall, J. and Ericsson, I. (1999) Chemical treatment of machined titanium surfaces. An in vitro study. Clin. Oral Implants Res. 10, 204-211 